\title{
Análisis de riesgos para la reserva ecológica de América Latina y el Caribe
}

Estela Monica Lopez Sardi ${ }^{1}$ y Beatriz Noemí García de Chena²

\section{Resumen}

Pensar en América Latina es pensar en las playas del Caribe, las selvas Amazónicas o las grandes extensiones de campo de Argentina. Imaginamos abundancia de recursos naturales en amplios territorios donde conviven comunidades representativas de una gran diversidad cultural. En la actualidad, los países de la región tienen, en conjunto, superávit ecológico, esto quiere decir que la biocapacidad de América latina y el Caribe como conjunto, supera su huella ecológica. Debido a esto es frecuente expresar que Latinoamérica y el Caribe financian con sus servicios eco - sistémicos a otros países o regiones del planeta que son grandes deudores ecológicos. Los últimos reportes publicados a este respecto nos muestran que si bien muchos países de la región aún presentan un saldo o reserva ecológica de signo positivo, ya existen amenazas que pueden afectar seriamente este balance. Nuestro objetivo es analizar los factores que ponen en riesgo la reserva ecológica positiva que hasta ahora ha caracterizado a América Latina y considerar propuestas de acciones tendientes su sostenibilidad en el tiempo.

Palabras clave: América Latina y el Caribe, huella ecológica, reserva ecológica.

1 Profesora Titular de Química General I (EST) y Química General (UP). Facultad de Ingeniería de la Universidad de Palermo y Escuela Superior Técnica del Ejército Grl Div Manuel N. Savio, Argentina.

2 Profesora Titular de Química General II (EST). Escuela Superior Técnica del Ejército Grl Div Manuel N. Savio, Argentina. 


\section{Abstract}

Think about Latin America is to think about the Caribbean beaches, the Amazon jungle or the large fields of Argentina. We imagine abundance of natural resources and biodiversity living in large areas and a number of communities of great cultural diversity. The región has an historically large ecological surplus. This positive situation is beginning to see threats that can seriously affect its balance so far favorable. The aim of this paper is to analyze the risks that threaten the positive ecological reserve that has hitherto sustained Latin America and consider actions for its sustainability.

Keywords: Latin America and the Caribbean, ecological footprint, ecological reserve. 


\section{Introducción}

\section{Huella ecológica}

La Huella ecológica fue definida en 1996 por William Rees y Mathis Wackernagel (Universidad de la Columbia Británica) como: "El área de territorio ecológicamente productivo (cultivos, pastos, bosques o ecosistema acuático) necesaria para producir los recursos utilizados y para asimilar los residuos producidos por una población definida con un nivel de vida específico indefinidamente, donde sea que se encuentre esta área."

Se trata de un indicador sencillo de comprender y de gran potencia comunicacional. Se puede aplicar a distintas escalas: personal, municipal, de países, mundial. Se trata de una herramienta nueva cuyos métodos de cálculo se encuentran en proceso de refinación donde la principal dificultad para el cálculo es generalmente la dificultad de obtener información por parte de algunos niveles gubernamentales.

La unidad de superficie utilizada para su medición es la hectárea global (hag). Como la productividad del terreno varía notoriamente de un lugar a otro, la hectárea global es una hectárea de terreno cuyo coeficiente de productividad es el valor medio mundial. La premisa de partida para el cálculo de la huella ecológica es que todos los procesos productivos, los consumos de materiales y energía y la absorción de los residuos tienen su correlato en una determinada superficie de territorio productivo necesaria para su producción o eliminación.

Los terrenos o superficies a tener en cuenta para el cálculo son:

- Terreno necesario para cultivos

- Terreno necesario para pastoreo de ganadeo

- Superficies forestales, bosques naturales o repoblados que se encuentren en explotación.

- Superficie de mar productivo, asociado a la actividad humana.

- Áreas de terreno urbanizadas.

- Área de bosques para absorción de $\mathrm{CO}_{2}$.

- Superficies asociadas al consumo energético, producción industrial, producción de servicios, transporte y comunicaciones.

- Superficie requerida para la conservación de la biodiversidad planetaria.

\section{Huella ecológica mundial}

La huella ecológica mundial se define como la superficie ecológicamente productiva (tierra o agua), requerida para proporcionar todos los recursos, bienes y servicios ecológicos que demanda la humanidad y para asimilar los residuos generados. Ambos procesos de generación y de asimilación compiten por el espacio. 
Biocapacidad es la capacidad de un sistema para producir recursos, bienes y servicios ecológicos y para absorber los desechos resultantes de su consumo. (Rosso, 2010, párr. 2). Ambos indicadores se miden en hag (hectáreas globales), generalmente expresadas per cápita (hagpc), para permitir comparaciones y el estudio de su evolución.

Los cálculos de huella ecológica mundial no son proyecciones especulativas sobre el futuro, sino una contabilidad del pasado y el presente, expresada en la cantidad de planetas Tierra necesarios para sostener el estilo de vida de la humanidad en un año determinado. La mejor evaluación de la evolución de la huella ecológica planetaria es la que realiza cada dos años WWF (World Wildlife Fund), publicación que se conoce como Informe Planeta Vivo (IPV). Según el Informe Planeta Vivo 2014 (WWF, IPV 2014, pág. 10), la huella ecológica mundial del planeta ha superado la biocapacidad planetaria, siendo necesarios los servicios ecológicos de 1,5 planetas Tierra para sostener el ritmo de vida de la humanidad durante un año. Por servicios ecológicos, naturales o eco - sistémicos (concepto desarrollado por el economista Robert Costanza y sus colaboradores) entendemos a todos aquellos servicios y recursos que nos proporciona la naturaleza que son indispensables para el funcionamiento de las sociedades y de los ecosistemas y a los cuales es difícil asignar un valor en términos monetarios de acuerdo con la economía clásica. Entre estos servicios podemos mencionar la generación de oxígeno, la captación de dióxido de carbono, la recarga de las napas acuíferas, la preservación del suelo y de la biodiversidad, entre muchos otros. En otras palabras: el planeta necesita 18 meses para regenerar los servicios naturales que consumimos en un año.

Según el mismo informe y en términos generales, la biocapacidad planetaria ha aumentado significativamente en términos de superficie (de 9900 millones a 12000 millones de hag entre 1961 y 2010), gracias al incremento productivo de la tierra derivado de los avances tecnológicos en riego y agricultura. Sin embargo, en forma paralela a este aumento, la población humana creció de 3100 a 7000 millones de habitantes, lo que hizo disminuir la biocapacidad media mundial per cápita de 3,2 hag a 1,7 hag. En el mismo período la huella ecológica media mundial aumentó de 2,5 a 2,7 hag per cápita. Con una perspectiva de superar los 9000 millones de habitantes en 2015 y los 11000 millones en 2100, la humanidad enfrenta un difícil desafío: lograr mayores aumentos de la biocapacidad terrestre pese a padecer problemas tales como la degradación del suelo, la escasez cada vez mayor de agua dulce y los elevados costos de la energía. (WWF, IPV 2014, pág. 11).

La huella ecológica (HE) se obtiene al sumar las huellas producidas por las distintas actividades antrópicas (Ecuación1).

HE $=$ Huella de carbono + Huella de cultivos + Huella forestal + Huella pesquera + Huella de pastoreo

Ecuación 1. Términos que conforman la huella ecológica. Fuente IPV, 2014. 


\section{Huella ecológica de un país}

La huella ecológica de una nación constituye actualmente una herramienta eficaz para realizar la contabilidad y administración de sus recursos naturales y un indicador que permite el diseño de políticas públicas ambientalmente sustentables.

Los principales factores relacionados con el crecimiento de la HE de un país son el incremento de la población y el aumento del consumo de bienes y servicios per cápita.

Actualmente, en los países de África, el crecimiento de la HE tiene origen únicamente en los aumentos de población. En América del Norte, América Latina, Oriente Medio, Asia Central y Asia del Pacífico, la HE crece impulsada por ambos factores (crecimiento poblacional e incremento del consumo per cápita) pero el principal impulso es el debido al crecimiento demográfico. En la Unión Europea ambos factores tienen incidencia similar en la HE y los Países Europeos no Comunitarios han experimentado una disminución de su HE debido a su decrecimiento demográfico.

Al ordenar los países según su HE total o su HE per cápita, se observan resultados muy diferentes. (WWF, IPV 2014, pág. 37).

Al analizar la huella ecológica de países respecto del valor global, un grupo de cinco naciones (China, Estados Unidos, Brasil, India y Rusia) son responsables del $47 \%$ de la HE total mundial (Fig. 1).

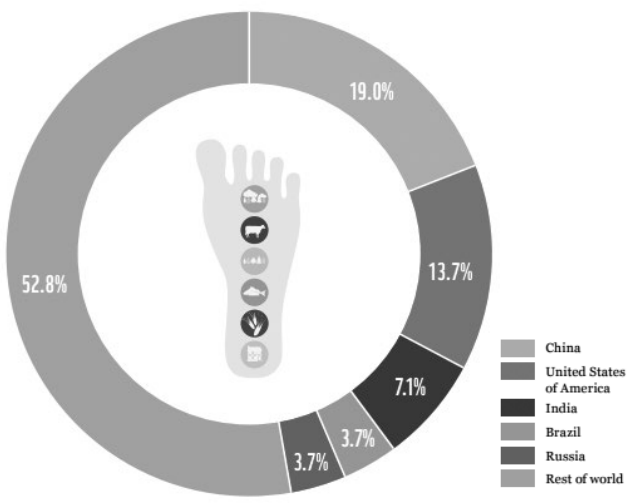

Figura 1. Huella Ecológica: Los cinco países top versus el resto del mundo. Fuente IPV 2014

La huella ecológica per cápita está relacionada con la cantidad de bienes y servicios consumidos por el habitante promedio de ese país. Por eso no es sorprendente que los 25 países con mayor HE per cápita sean naciones de altos ingresos, donde la HC es el principal componente de la HE. Tampoco sorprende que cambie significativamente el orden de aparición de los países al aplicar este criterio de clasificación. 
Debido a la gran magnitud de su población, China baja del primer lugar en HE total al puesto 76 en HE per cápita. Estados Unidos pasa del segundo lugar que ostentaba en la clasificación por HE total al séptimo lugar en la clasificación por HE per cápita. La India baja del tercer lugar al puesto número 136. El país con mayor huella ecológica per cápita a nivel mundial es Kuwait, superando las 10 hagpc. Lo siguen Qatar, Emiratos Árabes Unidos, Dinamarca y Bélgica.

Respecto de la biocapacidad mundial, el 60\% está distribuido en tan sólo 10 países del planeta: Brasil, China, USA, Rusia, India, Canadá, Indonesia, Australia, Argentina y República Democrática del Congo. Que la Argentina sea el noveno de esos 10 países privilegiados en el mundo es un gran orgullo pero sobre todo una grandísima responsabilidad.

\section{Reserva o saldo ecológico de un país}

El saldo ecológico de un país, de una región o del planeta, por lo general llamado reserva ecológica, se obtiene a partir del cálculo propuesto en la Ecuación 2.

$$
\begin{gathered}
\mathbf{R E}=\mathbf{B C}-\mathbf{H E} \\
\text { Ecuación } 2
\end{gathered}
$$

Siendo RE: reserva ecológica

BC: biocapacidad

HE: huella ecológica

La HE de una nación puede exceder su BC, operando en déficit ecológico al utilizar sus recursos y ecosistemas más rápido de lo que estos se regeneran. Una reserva ecológica de signo negativo es posible durante un cierto período, al utilizar recursos que se han acumulado a lo largo del tiempo. Otra manera de sostener una $\mathrm{HE}$ superior a la propia $\mathrm{BC}$ es importar productos de consumo, lo que equivale a exportar huella ecológica hacia otras naciones. Un país con elevada HE no solo afecta sus propias reservas ambientales, también tiene mayor incidencia en la degradación de los bienes globales comunes, por ejemplo, a través de la mayor cantidad de emisión de gases de efecto invernadero hacia la atmósfera.

Cuando un país presenta saldo positivo se dice que tiene superávit ecológico (es acreedor ecológico), mientras que un saldo negativo lo convierte en deudor ecológico.

A nivel planetario la humanidad como colectivo está en deuda con el ecosistema planetario. Como se dijo más arriba, la actual demanda mundial de recursos ecológicos renovables y de los bienes y servicios que la naturaleza nos provee 
equivalen a los suministrados por 1,5 planetas Tierra. Esta deuda ecológica es una enfermedad planetaria que ya empieza a manifestar sus primeros síntomas en forma de desertificación de grandes superficies, pérdidas de área de bosques y de selva, disminución del número de especies vegetales y animales (pérdida de biodiversidad), acumulación de gases de efecto invernadero y cambio climático.

\section{La situación de América Latina}

Cuando pensamos en América Latina, pensamos en las playas del Caribe, las selvas Amazónicas o las grandes extensiones de campo de Argentina. Imaginamos abundancia de recursos naturales, en amplios territorios donde conviven comunidades representativas de una gran diversidad cultural. Los países de la región tienen, en conjunto, superávit ecológico, esto quiere decir que su biocapacidad supera su huella ecológica. Debido a esto es frecuente expresar que Latinoamérica y el Caribe financian con sus servicios eco - sistémicos a otros países o regiones del planeta que son deudores ecológicos.

Los últimos reportes publicados a este respecto nos muestran que si bien el saldo ecológico positivo aún se mantiene en muchos países de la región, se empiezan a vislumbrar amenazas que pueden afectar seriamente este balance hasta ahora favorable.

Según la FAO (Food and Agriculture Organization of the United Nations), la región de América Latina y el Caribe comprende 46 países, territorios dependientes y departamentos de ultramar. La mayoría de los países están fuertemente urbanizados, con un $70 \%$ de su población viviendo en zonas urbanas, aunque muchas de ellas en estado de pobreza. Su territorio, muy dispar en lo que a características del suelo y el clima se refiere, puede ser dividido en diferentes zonas agroecológicas, en función del clima, la altura y el suelo: Trópico húmedo (gran parte de Brasil, Caribe y América Central), trópicos y subtrópicos semiáridos (México), trópicos y subtrópicos subhúmedos (Brasil, Colombia y Venezuela), tierras húmedas (parte de América Central y el cono sur: Argentina, Paraguay, Uruguay) y tierras en pendiente (Región Andina del cono sur, América Central y México). Se estima que el $25 \%$ de la población vive del fruto de las actividades vinculadas a la producción agrícola ganadera. La región presenta como características una distribución desigual de los recursos, grandes extensiones de territorio apto y no explotado, y predominancia de grandes unidades de organización como las estancias, haciendas y otros latifundios, en detrimento de la explotación de establecimientos más pequeños como las chacras y las granjas. (FAO, 1997, parte 2.1.6)

Debido a una organización económica basada principalmente en la producción de bienes provenientes del sector primario, los principales componentes de la huella 
ecológica de los países latinoamericanos son la huella derivada de los cultivos, la huella del pastoreo del ganado y la huella de la explotación forestal.

La Fundación Avina, que desde 1994 trabaja en América Latina y el Caribe creando alianzas entre la empresa privada, las instituciones filantrópicas y los estamentos gubernamentales para impulsar el desarrollo sostenible, detalla en su informe sobre sustentabilidad climática que “...América Latina cuenta con un tercio de las reservas de agua dulce, $12 \%$ de la superficie cultivable y $21 \%$ de la superficie de bosques naturales del mundo. Alberga los arrecifes coralinos más largos del hemisferio occidental y el Bioma Amazónico es emblema global de la mitigación y adaptación al cambio climático. El bioma provee bienes y servicios ambientales clave, como la regulación regional y global del clima, la reserva de 2/3 de la biomasa forestal del mundo y una alta riqueza biológica, recursos genéticos y medios de vida. Además, posee una identidad cultural diversa, con más de 400 pueblos indígenas viviendo en la región.” (Fund. Avina, 2013, párr. 1). Esta capacidad de proveer servicios eco - sistémicos coloca a la región en una situación privilegiada frente al futuro.

Un análisis de la situación país por país, permite observar que la reserva ecológica en muchos casos presenta saldo negativo, sin bien el saldo global regional aún resulta ser positivo. En la Tabla 1 se detalla la situación de gran parte de los países de la región, comparando la biocapacidad per cápita del país respecto de su huella ecológica per cápita.

\begin{tabular}{|l|c|c|c|}
\hline País & Bc (hagpc) & He (hagpc) & Re (hagpc) \\
\hline Argentina & 8,2 & 2,5 & 5,7 \\
\hline Bolivia & 15,7 & 2,1 & 13,6 \\
\hline Brasil & 7,3 & 2,4 & 4,9 \\
\hline Chile & 4,1 & 3 & 1,1 \\
\hline Colombia & 4,1 & 1,9 & 2,2 \\
\hline Costa Rica & 1,9 & 2,3 & $-0,4$ \\
\hline Cuba & 1,1 & 1,8 & $-0,7$ \\
\hline Rep. Dominicana & 0,8 & 1,5 & $-0,7$ \\
\hline Ecuador & 2,1 & 2,2 & $-0,1$ \\
\hline El Salvador & 0,7 & 1,6 & $-0,9$ \\
\hline Guatemala & 1,3 & 1,5 & $-0,2$ \\
\hline Haití & 0,2 & 0,5 & $-0,3$ \\
\hline Honduras & 1,9 & 1,8 & 0,1 \\
\hline Jamaica & 0,6 & 1,1 & $-0,5$ \\
\hline México & 2 & 3,4 & $-1,4$ \\
\hline Nicaragua & 3,2 & 2 & 1,2 \\
\hline Panamá & 9,5 & 3,2 & 6,3 \\
\hline Paraguay & 9,7 & 3,2 & 6,5 \\
\hline
\end{tabular}




\begin{tabular}{|l|c|c|c|}
\hline Perú & 4,1 & 1,6 & 2,5 \\
\hline Uruguay & 10,5 & 5,5 & 5 \\
\hline Venezuela & 3,1 & 2,8 & 0,3 \\
\hline TOTAL REGIONAL & $\mathbf{9 2 , 1}$ & $\mathbf{4 7 , 9}$ & $\mathbf{+ 4 4 , 2}$ \\
\hline
\end{tabular}

Tabla 1. Reserva Ecológica de países de América Latina y el Caribe. Fuente: Global Footprint Network (2008)

Al analizar la Tabla 1, llama la atención el caso de Costa Rica, país reconocido a nivel mundial en su calidad de gran proveedor de servicios eco - sistémicos, cuya saldo o reserva ecológica es, sin embargo, negativo. Un estudio de la Universidad de Costa Rica, que analiza las principales causas de la elevada HE del país, destaca los siguientes factores: alta huella de carbono, asociada principalmente a la producción de energía, emisiones de $\mathrm{CO} 2$ y ruidos provenientes de la flota vehicular, elevado consumo de agua potable per cápita, muy por encima del promedio mundial y bajo grado de tratamiento de las aguas residuales, provocando la contaminación de esteros, ríos y el litoral marino.

Los datos numéricos sitúan a Uruguay como el país de mayor huella ecológica per cápita de América Latina, reflejo de su producción asociada a los recursos naturales y a su reducida población. El informe sobre Desarrollo Humano 2011 del PNUD (Programa de las Naciones Unidas para el Desarrollo) enumera las siguientes razones como responsables de esta circunstancia:

- Deterioros asociados a la actividad agropecuaria (que ocupa el $90 \%$ del territorio de Uruguay): emisiones, erosión y degradación de la tierra, pérdida de biodiversidad y afectación de los recursos hídricos.

- Deterioros asociados a la industria turística, que generan una fuerte presión sobre los ecosistemas costeros.

\section{Riesgos y amenazas}

\section{Cambio climático}

Según el IPCC (International Pannel of Climate Change), la variabilidad climática ya es observable en América Latina, registrándose aumento en la frecuencia de fenómenos tales como fuertes inundaciones en Brasil, Colombia, Uruguay, Argentina y Venezuela, de huracanes en el Caribe y de sequias en Amazonia. También se observa mayor frecuencia de incendios forestales y disminución superficial de los glaciares andinos. (IPCC, 2007, cap.13) 
El Banco Internacional de Desarrollo estima que Latinoamérica sufrirá para 2050 pérdidas del orden de los 100 billones de dólares vinculadas a la disminución de sus exportaciones agrícolas por causa del cambio climático. (2014, BID Executive Summary, párr. 3).

\section{Salud Pública}

Según CEPAL el cambio climático provocará la ampliación del territorio afectado por enfermedades cuyo vector es el mosquito, cuyas especies son sensibles a los cambios de temperatura. Entre estas enfermedades se encuentran el dengue, la malaria y la fiebre amarilla. Un ejemplo de ello es el Aedes aegypti, que en 1970 se encontraba solamente en Venezuela, Surinam, Guayana y el Caribe y desde 2002 sólo ciertas regiones ubicadas muy al sur del continente se encuentran libres de su presencia. (CEPAL, 2010, pág. 20).

Otro ejemplo lo constituye la fiebre chikungunya, originaria de África y Asia, transmitida por mosquitos y detectada en América desde 2013. (2014, Ministerio de Salud, párr. 2)

\section{Energía}

Uno de los puntos clave para el crecimiento económico es la provisión de energía. Una de las fortalezas de América Latina ha sido una matriz energética mayoritariamente limpia, con gran producción hidroeléctrica y con su principal economía (Brasil) situada como líder mundial en la producción de biocombustibles. (Fund. Avina, 2013, párr. 5). Sin embargo, el descubrimiento de grandes yacimientos de gas y petróleo, muchos de ellos explotables mediante la tecnología de fraccionamiento hidráulico (fracking), sumado a la actual baja internacional del precio del petróleo, amenazan el fortalecimiento de una matriz energética limpia y eficiente. Según la Organización latinoamericana de Energía (OLADE), América Latina cuenta con el $23 \%$ de las reservas mundiales de petróleo.

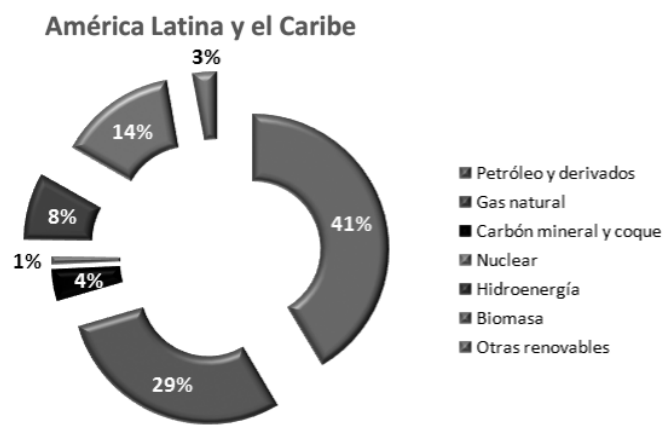

Figura 2. Matriz energética de América Latina. Fuente OLADE 2012. 
En la Figura 2 se puede observar la fuerte dependencia regional de los combustibles fósiles como fuente de energía. El desafío regional es lograr un crecimiento de la matriz energética que favorezca el despegue económico y el bienestar, pero dando mayor prioridad a la instalación de tecnologías de energía limpia.

\section{Actividad económica}

La magnitud de cada uno de los componentes de la huella ecológica de un país es proporcional a las actividades en las que basan su economía.

Mientras que el componente Huella de carbono (huella derivada de la industrialización y el consumo) de la Huella ecológica en América Latina se encuentra por debajo de la media mundial, la huella que proviene del uso del suelo es muy superior a la del resto del mundo. Este uso del suelo está muy relacionado con la estructura económica de la región, donde las actividades más desarrolladas son las agrícola - ganaderas. La huella provocada por la deforestación en América Latina es la más alta a nivel mundial.

Un ejemplo drástico de la influencia de la actividad económica en el balance ecológico lo constituye el caso de Uruguay antes descripto, país que desde hace casi cinco años ostenta la mayor huella ecológica de la región, pese a ser un país preocupado por las políticas verdes y el cuidado ambiental.

La minería es otra actividad en crecimiento en América Latina. La producción de metales no ferrosos aumentó de 2000 a 18000 millones de dólares entre 2003 y 2013. La incidencia de la minería en el PIB supera el 10\% en países como México, Bolivia, Colombia y Perú (Minería Panamericana, 2013, párr. 1). La actividad minera conlleva altos riesgos de carácter ambiental a los que se suman los conflictos sociales evidenciados en distintas zonas ante la posible instalación de algunas explotaciones mineras.

La tendencia histórica de las economías latinoamericanas de basar su crecimiento en la exportación de materias primas obtenidas por la explotación intensiva de sus recursos naturales sin valor agregado constituye uno de los principales riesgos a futuro para los ecosistemas regionales.

\section{Biodiversidad}

Según el Índice Planeta Vivo 2014, la caída más drástica en la biodiversidad durante el período 1970 - 2010 se observó en América Latina. Las causas principales son la pérdida de hábitats, los cambios en el uso del suelo y la explotación directa de especies a través de la caza y la pesca. Simultáneamente se ha observado una tendencia al aumento en la biodiversidad de los países ubicados en regiones más desarrolladas, como América del Norte y Europa. La explicación a este fenómeno 
se puede encontrar en la tendencia actual de los países desarrollados a importar gran parte de sus recursos básicos, exportando la pérdida de biodiversidad a países menos desarrollados cuyas economías se basan en la exportación de productos con bajo o nulo valor agregado. (IPV 2014, pág. 8).

\section{Análisis sintético}

Existe una relación causa efecto entre los riesgos y amenazas regionales y su influencia en la Huella Ecológica. Esta situación se sintetiza en el Diagrama de Ishikawa de la Figura 3.

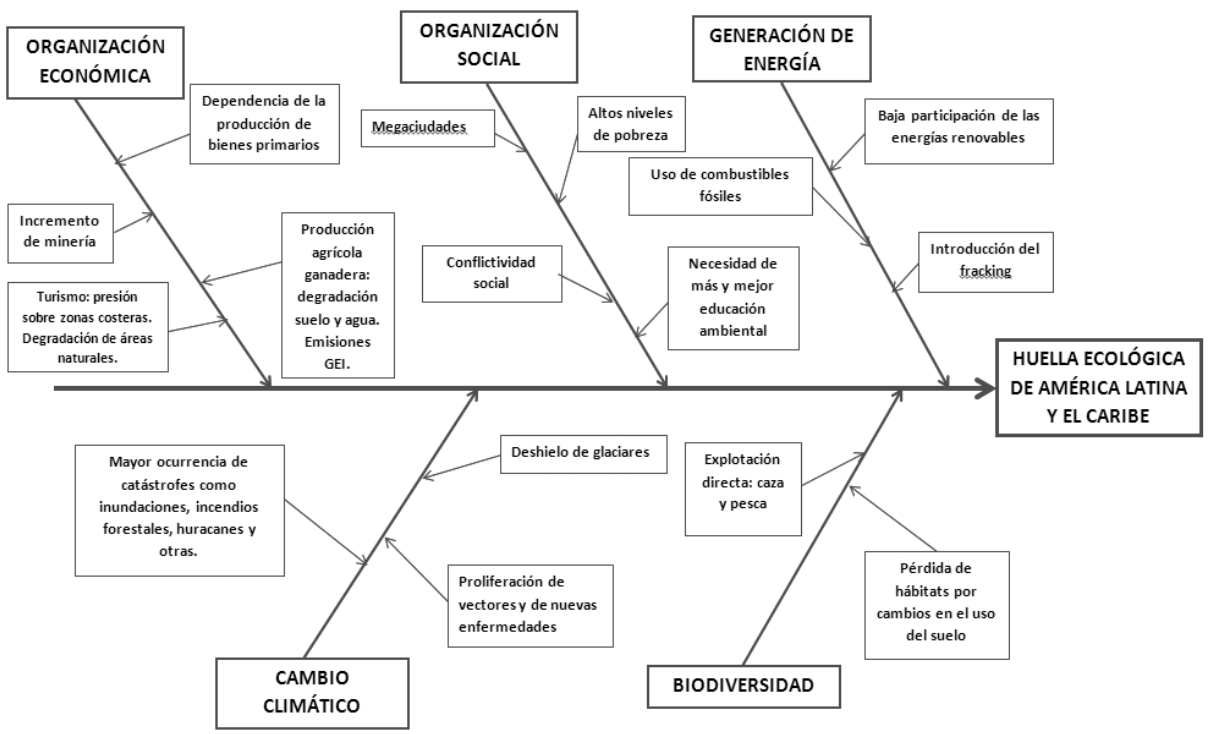

Figura 3. Factores que inciden en la huella ecológica regional.

\section{Desafíos y oportunidades}

América Latina y el Caribe todavía tienen crédito ecológico y son líderes a nivel mundial en la provisión de servicios ambientales tales como la captura de carbono. Tiene también vastas zonas que, de ser conservadas apropiadamente, constituyen un reservorio planetario de biodiversidad.

Según el Banco Mundial, en la última década el 50\% de la población de la región pasó a formar parte de la clase media. Pero mientras que setenta millones de personas salieron de la pobreza, aún hay 130 millones de personas que no logran dar ese salto, viviendo en condiciones de pobreza crónica. El crecimiento 
regional del último año se vio afectado por la desaceleración de las economías de Argentina y Brasil. (BM, 2014, párr. 5). El desafío de lograr en los próximos años un desarrollo económico regional que se traduzca en un mayor bienestar de la población con mínimo impacto ambiental es una meta que requiere cuidadosa planificación. Requiere la introducción de los principios del desarrollo sustentable en las políticas y programas nacionales, para reducir las pérdidas de recursos naturales y biodiversidad en el camino al crecimiento. Es imprescindible también el fortalecimiento de organizaciones de la sociedad civil, cuyas actividades de denuncia ante las decisiones de los sectores público y privado obligue a una mayor transparencia en la toma de decisiones (Leef et al, 2001, pág. 25).

En la Tabla 2 se sintetizan las fortalezas, debilidades, oportunidades y desafíos ambientales para América latina y el Caribe.

\section{FORTALEZAS:}

Riqueza en climas y paisajes. Biodiversidad. Abundancia de agua potable.

Abundancia de tierras productivas y de recursos renovables y no renovables.

Gran diversidad cultural.

Superávit ambiental.

\section{OPORTUNIDADES:}

Desarrollar las oportunidades en el campo de los servicios ambientales.

Desarrollar y apoyar modelos de negocios ambientalmente sostenibles.

Captación de recursos financieros para mecanismos de desarrollo limpio. (MDL).

Desarrollo con igualdad.

\section{DEBILIDADES:}

Dependencia económica de las actividades del sector primario.

Baja conciencia ambiental en los estratos gubernamentales.

Desigualdad social.

Deficiencias en la educación en general y en la educación ambiental en particular.

\section{AMENAZAS:}

Presión humana en ciudades y megaciudades como Bs. As. Río de Janeiro, San Pablo, México y otras.

Conflictividad social.

Acumulación de residuos.

Catástrofes atribuibles al cambio climático.

Carencia de políticas ambientales

gubernamentales sostenidas en el tiempo.

Tabla 2. América latina y el Caribe. Análisis FODA.

\section{Algunas propuestas}

A continuación se sintetizan algunas de las principales estrategias propuestas para la región, con el fin de conservar el patrimonio ambiental:

- Protección del capital natural, aumentando la red de áreas protegidas, deteniendo la pérdida de hábitats prioritarios y promoviendo la restauración de los ecosistemas dañados. 
- Promover y aumentar la producción de energía renovable.

- Mejorar la producción de bienes y servicios, gestionando los recursos de forma sostenible, reduciendo los insumos y disponiendo eficientemente de los desechos.

- Educar bajo nuevos modelos, promoviendo el consumo responsable y estilos de vida de baja huella ecológica.

- Reorientar los flujos financieros, promoviendo la puesta en valor de la naturaleza, contabilizando los costos ambientales y sociales de los emprendimientos económicos.

- Recompensar las acciones de innovación y gestión sostenible de recursos.

- Establecer planes de gobernanza que contemplen una distribución equitativa de los recursos, favoreciendo las opciones ecológicas y socialmente justas. Modelo del desarrollo con igualdad.

- Elegir indicadores de éxito en la gestión gubernamental de las naciones que se encuentren "más allá del PBI".

- Impulsar la comercialización preferencial de productos con valor agregado que cumplan con requisitos ambientales.

- Apoyar e impulsar los mecanismos de desarrollo limpio (MDL).

\section{Conclusión}

Lograr avances en la implementación de estas medidas requiere cooperación y acuerdos transnacionales. En un momento histórico en que los gobiernos de la región buscan afianzar la unidad geopolítica, sería importante que los gobernantes colocaran en un lugar preeminente de la agenda los tópicos vinculados a la preservación eco - sistémica de América Latina y el Caribe.

Ningún indicador ni métrica ambiental puede captar en su totalidad un sistema tan complejo como nuestro planeta. Sin embargo, indicadores como la huella ecológica y la reserva ecológica de los países y regiones pueden resultar herramientas claras de análisis de la situación pasada y presente, útiles para evaluar los logros alcanzados y para diseñar las estrategias que nos acerquen a la meta de la sostenibilidad ambiental. 


\section{Referencias}

Banco Mundial (2014) América latina y el Caribe: panorama general. Obtenido el 17 de abril de 2015 en http://www.bancomundial.org/es/region/lac/overview

CEPAL (2010). Gráficos Vitales del Cambio Climático para América Latina y El Caribe. Obtenido el 17 de abril de 2015 en http://www.cepal.org/dmaah/ noticias/paginas/7/37867/LAC_Web_esp_2010-12-02.pdf

Dávalos, V. O. (2012). Matriz Energética en América Latina y El caribe. Situación actual y perspectivas de las energías renovables. La Habana. Cuba. Obtenido el 30/04/2015 en: http://www.olade.org/sites/default/files/presentaciones-sej/8_ Presentaci\%C3\%B3n\%20OLADE\%20UPADI\%20201.pdf

Data Sources. (2008) Global Footprint Network. Obtenido el 17-04-2015 en http://es.wikipedia.org/wiki/Anexo:Pa\%C3\%ADses_seg\%C3\%BAn_su_ huella_ecol\%C3\%B3gica.

Era Verde. (2012). Costa Rica en Rojo con su Huella Ecológica y el Estado es parte del problema. Universidad de Costa Rica. Obtenido el 30-04-2015 en: http:// www.eraverde.ucr.ac.cr/blog/costa-rica-en-rojo-con-su-huella-ecologica-y-elestado-es-parte-del-problema

Slekis Riffel, G. (2012). Humanismo Uruguayo. Informe sobre huella ecológica. Obtenido el 30/04/15 en: https://humanismouruguayo.wordpress. com/2012/06/20/informe-sobre-huella-ecologica/

Fundación Avina, (2013) 2014, Año clave para que Latinoamérica incida en la sustentabilidad climática. Obtenido el 8 de abril de 2015 en http://www.avina. net/esp/8769/incontext-19/

Banco Internacional de Desarrollo. IDB. (2014). Executive Summary: The Climate and Development Challenge for Latin America and the Caribbean: Options for Climate Resilient Low Carbon Development. Obtenido el 17 de abril de 2015 en http://idbdocs.iadb.org/WSDocs/getDocument.aspx?DOCNUM=36898600

International Pannel of Climate Change. IPCC. (2007). Climate Change 2007: Impacts, Adaptation and Vulnerability. (last updated 15/4/2013). Obtenido el 14 de abril de 2015 en http://www.ipcc.ch/publications_and_data/publications ipcc_fourth_assessment_report_wg2_report_impacts_adaptation_and_ vulnerability.htm 
Leef, E.; Bastida, M. (2001). Comercio, medio ambiente y desarrollo sustentable: perspectivas de américa latina y el caribe. UNAM. Obtenido el 17 de abril de 2015 en http://www.ambiente.gov.ar/infotecaea/descargas/leff07.pdf

FAO. (1997). Lista Mundial de Vigilancia para la Diversidad de los Animales Domésticos. (2a Edición) Scherf B. (Ed.). FAO. UNEP. Organización de las Naciones Unidas para la agricultura y la alimentación. Obtenido el 8 de abril de 2015 en http://www.fao.org/docrep/v8300s/v8300s00.htm\#Contents

Minería Panamericana (2013) América Latina: epicentro de la inversión minera mundial. Obtenido el 20 de abril de 2015 en http://www.cpampa.com/web/ mpa/2013/05/america-latina-epicentro-de-la-inversion-minera-mundial/

Ministerio de Salud. Presidencia de la Nación. (2014) Fiebre chikungunya. Otenido el 17 de abril de 2015 en http:/www.msal.gov.ar/index.php/component/ content/article/48-temas-de-salud-de-la-a-a-la-z/441-fiebre-chikungunya-

Navarra, E. (2012). Huella ecológica y sostenibilidad. Obtenido el 30 de abril de 2015 en http://www.cfnavarra.es/medioambiente/agenda/Huella/Ecosos.htm

ONU para la alimentación y la agricultura. (2013). Agro noticias para América latina y el Caribe : América Latina y la sustentabilidad ecológica. Obetnido el 8 de abril de 2015 en http://www.fao.org/agronoticias/agro-noticias/detalle/ es/c/210863/

Rosso, A. (2010) ¿Cuán grandes son nuestros pies? Programa Huella Ecológica y Biocapacidad Argentina. INTI. Obtenido el 20 de abril de 2015 en http://www. inti.gob.ar/sabercomo/sc95/inti9.php

WWF. (2012) Informe Planeta Vivo 2012. WWF Internacional, Gland, Suiza. Obtenido el 20 de abril de 2015 en http:/awsassets.wwfar.panda.org/ downloads/informe_planeta_vivo_2012.pdf

WWF. (2014). Informe Planeta Vivo 2014: Personas y lugares, especies y espacios. McLellan, R., Iyengar, L., Jeffries, B. and N. Oerlemans (Eds), WWF Internacional, Gland, Suiza. Obtenido el 7 de abril de 2015 en http:// www.footprintnetwork.org/images/article_uploads/InformePlanetaVivo2014_ LowRES.pdf 